\title{
Making High-Performance Robots Safe and Easy to Use For an Introduction to Computing
}

\author{
Joseph Spitzer, ${ }^{1}$ Joydeep Biswas, ${ }^{2}$ Arjun Guha ${ }^{1}$ \\ University of Massachusetts Amherst ${ }^{1}$, University of Texas at Austin ${ }^{2}$
}

\begin{abstract}
Robots are a popular platform for introducing computing and artificial intelligence to novice programmers. However, programming state-of-the-art robots is very challenging, and requires knowledge of concurrency, operation safety, and software engineering skills, which can take years to teach. In this paper, we present an approach to introducing computing that allows students to safely and easily program highperformance robots. We develop a platform for students to program RoboCup Small Size League robots using JavaScript. The platform 1) ensures physical safety at several levels of abstraction, 2) allows students to program robots using JavaScript in the browser, without the need to install software, and 3) presents a simplified JavaScript semantics that shields students from confusing language features. We discuss our experience running a week-long workshop using this platform, and analyze over 3,000 student-written program revisions to provide empirical evidence that our approach does help students.
\end{abstract}

\section{Introduction}

Robots are frequently used to introduce computing to novice programmers (Osborne, Thomas, and Forbes 2010; Featherston et al. 2014; Magnenat et al. 2014; Dee et al. 2017; Gucwa and Cheng 2017; Musicant, Laddha, and Choi 2017; Paramasivam et al. 2017; Doran and Clark 2018; Paspallis et al. 2018). Since they are a hands-on medium, it is relatively easy to make an engaging robotics-based curriculum. Moreover, robots are a natural platform to introduce a variety of core STEM subjects, including geometry, mechanics, and programming. However, many robotics-based curricula use robots that are designed for education, and do not represent the state-of-the-art in robotics (Osborne, Thomas, and Forbes 2010; Featherston et al. 2014; Magnenat et al. 2014; Dee et al. 2017; Gucwa and Cheng 2017; Musicant, Laddha, and Choi 2017; Estrada 2017; Doran and Clark 2018; Paspallis et al. 2018).

Our research group maintains a team of soccer-playing robots that compete in the RoboCup Small Size League (SSL) tournament (Weitzenfeld et al. 2015). We designed, built, and programmed these robots ourselves, and wanted to use them in an outreach workshop for several reasons.

Copyright (C) 2020, Association for the Advancement of Artificial Intelligence (www.aaai.org). All rights reserved.

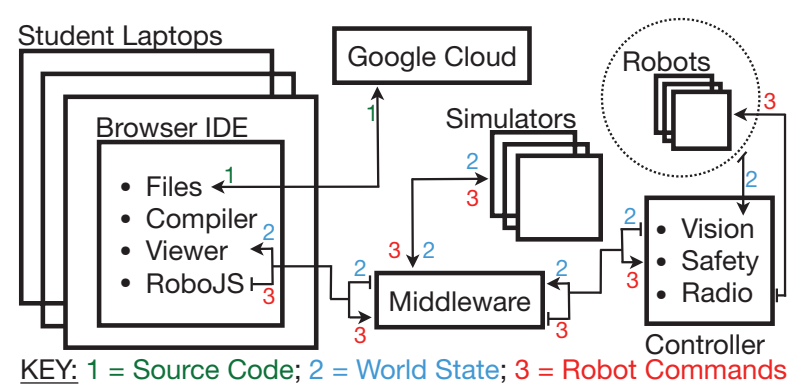

Figure 1: A schematic representation of the major components and data flow in our system.

1) SSL robots are compelling because they exhibit highperformance behaviors and demonstrate what state-of-theart robots can do. For example, they are omnidirectional, move at high speeds, and can manipulate a ball rapidly. 2) We can explain and demonstrate how the robots were built and the equipment needed to do so, namely CNC mills and CAD tools. 3) SSL robots are designed to compete in teams, thus students can use them to write cooperative and competitive agents for soccer and other activities (e.g. robot tag or navigation in a maze). 4) Soccer is a well-known domain.

However, high-performance robots such as ours, are not well-suited for novice programmers. 1) If programmed incorrectly, the robots can cause injury because they are able to move very rapidly. 2) We program the robots using a sophisticated low-level C++ API that lends itself to writing high-performance code, but requires significant expertise to use. 3) For competitive play, the robots employ a multi-tier abstraction for high-level team-wide planning and low-level control that continuously updates the robots' roles (Browning et al. 2005), which makes it difficult to issue simple movement commands to an individual robot.

Contributions The diagram in Figure 1 depicts the design of our system, which addresses these challenges.

1. We develop a robot control platform that checks for safety at several layers of abstraction, which allows novices to safely work with high-performance RoboCup SSL robots. 
2. We develop a simplified programming stack for controlling robots over the network (Middleware in Figure 1). This programming stack is resilient to transient network faults and provides simple interfaces to low-level robot motion controllers that provide functionality like grid navigation and ball interception.

3. Although we use a professional programming language, and not a programming language designed for education, we take several measures to shield students from complex and confusing behavior that the language normally exhibits. Specifically, we use JavaScript and build a source-to-source compiler that simplifies the semantics of JavaScript to make it more intuitive.

4. We develop a curriculum for a week-long outreach workshop that gradually introduces students to progressively more advanced mathematics and more sophisticated forms of robot control. Our robot programming APIs have multiple layers of abstraction that support this curriculum design.

We discuss our experience running a week-long workshop using this platform, and analyze over 3,000 program revisions created over the course of the workshop to argue that our approach does help students.

The rest of this paper is organized as follows. §2 reviews related work. $\$ 3$ presents our robot programming platform and our approach to safety. $\S 4$ presents our JavaScript programming environment, and the approach we use to simplify JavaScript for novice programmers. $\$ 5$ presents our workshop curriculum. $\$ 6$ presents evidence that our programming environment helps students catch bugs. Finally, $\S 7$ discusses future work and concludes.

Our software can be found on GitHub within the ut-amrl/robo-js repository.

\section{Related Work}

Young children exposed to basic computer science concepts with robots exhibit promising levels of comprehension by way of such a hands-on medium (Magnenat et al. 2014; Martinez, Gomez, and Benotti 2015). These sessions-and others including (Featherston et al. 2014)—use a block-based programming language alongside a simplified robotics platform. Scratch ${ }^{1}$, Alice ${ }^{2}$, and Blockly ${ }^{3}$ are some of the most common block-based programming languages. Lego Mindstorms $^{4}$, Thymio ${ }^{5}$, and Linkbot ${ }^{6}$ are popular educational robots; for each there is support of both block and text-based programming, either natively or through a third-party.

In contrast to other outreach workshops and exploratory activities within the high-school age range (Osborne, Thomas, and Forbes 2010; Musicant, Laddha, and Choi 2017; Paspallis et al. 2018), our students do not build or

\footnotetext{
${ }^{1}$ https://scratch.mit.edu

${ }^{2}$ https://www.alice.org

${ }^{3}$ https://developers.google.com/blockly

${ }^{4}$ https://www.lego.com/mindstorms

${ }^{5}$ https://www.thymio.org

${ }^{6}$ https://www.barobo.com
}

augment robotics hardware. All our robots were created independently; on-site setup and maintenance was performed by the workshop instructors.

Our formalization of a layer-based abstraction appears to be a novel design for short-term computer science outreach. There is an analogous approach outlined on a much different scale; a university curriculum (Doran and Clark 2018). Students begin with Java in CS1 using Lego Mindstorms by way of leJOS ${ }^{7}$, a Java based replacement for the existing Lego firmware. In future courses, native Lego hardware is replaced with an Arduino and Raspberry Pi for use of common libraries in C, Java, and Python.

Using simulation in conjunction with physical robots is quite common. Industrial grade simulation, provided by Gazebo $^{8}$, is excessive for introductory use as it can easily overwhelm students. For this reason, simulators wherein the target audience is users new to CS have been developed into products such as Robot Virtual Worlds ${ }^{9}$ and RoboSim ${ }^{10}$. The latter can be used seamlessly with physical robots (Gucwa and Cheng 2017). They outline ideal principles of a simulated environment, including uniform code and accurate real-world representation.

We use a modification of the standard vision system (Zickler et al. 2014) of RoboCup SSL. There exists an implementation of a similar lower-end system to interface with Lego Mindstorms using a web-cam and sending commands over Bluetooth (Estrada 2017).

To our knowledge, the most similar work to date is that done by a group from the University of Washington (Paramasivam et al. 2017). They conducted a week-long outreach workshop for high school students using JavaScript. Providing a small API for robot control, students wrote programs in a browser-based editor for a TurtleBot ${ }^{11}$. Perhaps the biggest difference was the robots that were used. The standalone TurtleBots with a touchscreen and speech input-output supplied a platform for programs centered around human-robot interaction. By contrast, our RoboCup SSL robots are in use simultaneously from the afternoon of the first day, thus activities are rather focused on multi-agent systems.

RoboCup serves as a challenging test-bed for several research problems, including failure recovery (Holtz, Guha, and Biswas 2018), time-optimal control (Balaban, Fischer, and Biswas 2018), and multi-agent planning (Biswas et al. 2014). In particular, RoboCup Jr. (Kitano, Suzuki, and Akita 2000) serves as a bridging league where high-school students can compete at international RoboCup competitions. Our work continues the tradition of using RoboCup to inspire the future generation of robotics by catering specifically to students via introduction to computing workshops.

\section{RoboCup Robots for Novice Roboticists}

We present our state-of-the-art soccer-playing robots and the associated software platform, including the existing de-

\footnotetext{
${ }^{7}$ http://www.lejos.org

${ }^{8} \mathrm{http}: / /$ gazebosim.org

${ }^{9} \mathrm{http}: / /$ www.robotvirtualworlds.com

${ }^{10} \mathrm{https}: / / \mathrm{c}-$ stem.ucdavis.edu/studio

${ }^{11}$ https://www.turtlebot.com
} 


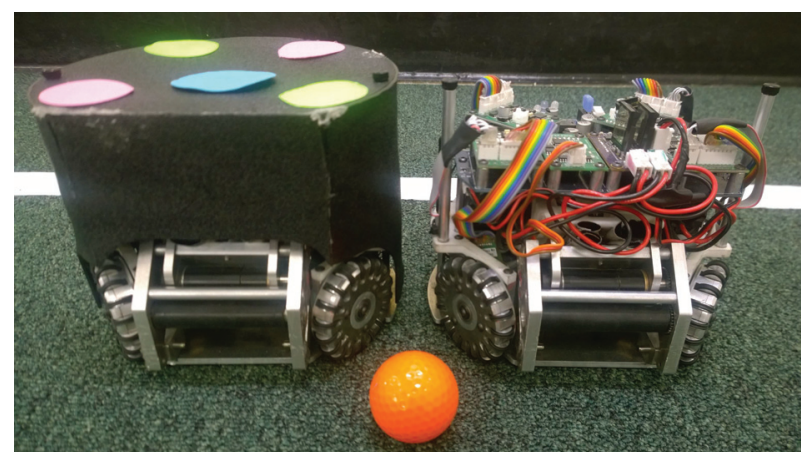

Figure 2: One of the robots alongside another with its casing removed. A colored golf ball is used as the ball.

sign and the necessary changes we made for the workshop. These include increased safety measures, middleware to tie the low-level robotics hardware layer with the end-user environment, and student access to our auxiliary simulator.

\subsection{Hardware Platform}

Figure 2 shows our custom-designed robots that we built to compete in the RoboCup SSL. The robots fit within a cylinder of $18 \mathrm{~cm}$ in diameter and are $15 \mathrm{~cm}$ tall. The electronics system is modular with the main circuit board handling receiving and processing commands as well as power distribution. Auxiliary boards control wheel motors and kick behavior. Kicking is accomplished by accelerating a ferromagnetic kicking rod using a custom-wound electromagnet with energy stored in a bank of capacitors. One of the immediate fascinations of these robots is that their movement is omnidirectional, and they are capable of moving precisely at high speeds while manipulating the ball in motion.

All robots move within a calibrated field, which is simply a Cartesian plane, thereby permitting use of standard Euclidean geometry and algebra in calculations. The field dimensions at our venue were $2.4 \mathrm{~m}$ by $3.6 \mathrm{~m}$. An overhead camera can uniquely identify each robot by their top dot pattern (Zickler et al. 2014). This vision system can instantaneously observe robots and a ball on the field; positions and velocities are updated in real-time.

\subsection{Robot Software Stack}

The software platform is written in $\mathrm{C}++$, with networking done over UDP multicast. It is multi-threaded in order to support concurrent 1) state estimation to update the state of the world based on vision data, 2) control and planning to assign roles to agents and to compute safe collision-free control trajectories, as well as 3) collation and transmission of commands to the robots.

Multi-robot coordination and task allocation is performed using Skills-Tactics-Plays (STP) (Browning et al. 2005). Skills define low-level actions, which the physical robot can perform, such as movement or kicking. Tactics group skills and determine which one to execute at a given time. For example, a Goalie tactic will try to block the ball if the trajectory of the ball would land in its goal. Finally, Plays group tactics for synchronized activity among multiple robots. Examples of plays include Kickoff or Free-kick.

We add a single tactic named Marionette to the STP stack running on our soccer robots. This tactic is special in the sense that it simply acts on data received from outside the system, after applying safety checks. It has the skills to move, dribble, catch, block, and kick. These skills are sufficient for the workshop and have corresponding library methods for the end programmer to interface with. For some skills there are multiple library methods exposed to the students; see section 4.4. Given the nature of the Marionette, there is no need for plays as all robots only run this tactic.

Also, we republish the current world state to UDP so it may be consumed outside the platform. These two items; the addition of the Marionette tactic and the modification to expose system state were the two main changes we had to make. In this sense we leverage all existing capabilities of the system by providing a means to exchange data, through our application middleware. This middleware serves as the intermediary between the low-level $\mathrm{C}++$ code that runs on the robot controller and the high-level JavaScript code the students write in the browser.

\subsection{Robot Safety Measures}

Given these are high-performance robots it is important that safety be taken into account both for the students and the robots. As such, we employ the following safety measures:

1. Reduced Motion Model: To ensure safe gameplay on a reduced field size, we limited the maximum speed of the robots to $1 \mathrm{~m} / \mathrm{s}$ (the robots are capable of maximum speeds in excess of $4 \mathrm{~m} / \mathrm{s})$.

2. Crash Prevention Buffer: Each robot has a uniform safety margin that surrounds it, enforced by Dynamic Safety Search (Bruce and Veloso 2006). This margin accounts for multi-robot dynamics and prevents robots from running into each other, even if the student-written programs erroneously command them to do so.

3. Command Timeouts: The robots have a command timeout such that if the robot control stack does not receive any commands from the student programs within a sliding five second window, the control stack aborts any active actions and brings the robots to a halt. Execution resumes seamlessly with the next command received.

4. Robot Identification Number Before any robot commands may be issued, the programmer must explicitly set the number of a robot that is presently available for control, thus disallowing the program from commanding robots that are not on the field.

5. Field Boundaries If a statement in a student's program erroneously instructs the robot to go beyond the field boundaries then the Marionette tactic truncates the commanded locations to allowable value(s).

6. Skill-based Checks The Marionette tactic verifies that the low-level skill commanded by the student program is indeed applicable based on the current world state - this allows it to catch errors where the student program for 


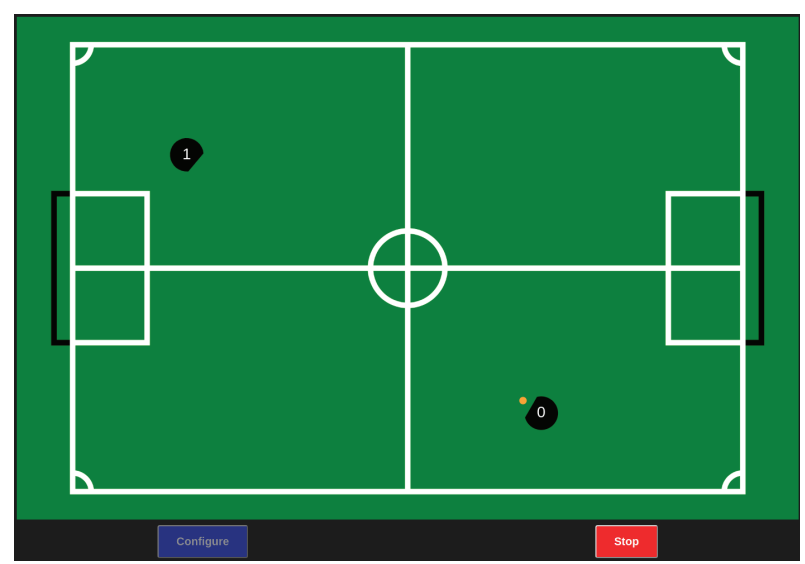

Figure 3: Two robots passing in simulation.

example tries to kick the ball when it is far from the robot or not in front of the robot.

\subsection{A Simulation Viewer in the Browser}

We provide a web-based viewer for corresponding simulators hosted on remote servers. The viewer displays the world state, relayed to it by way of the middleware intermediary in real-time. Beneath the visual pane are basic configuration options. Based on the programming activity, the students can select initial simulator conditions from a supplied list. Such items vary the number of robots, the ball and robot positions, and in some cases the background (e.g. soccer pitch or a maze). Some configurations involve randomization, so to provide a better means for program testing. Figure 3 shows our viewer while a simulation is running; the animations are $2 \mathrm{D}$ from a top-down perspective and are made using the HTML Canvas.

The choice to provide simulator viewing and control in the browser is twofold. For one it allows us to easily abstract away the exhaustive detail of the native viewer and the rigid command-line interface of the simulator. Also, by using the browser, the need for installation and hardware requirements is alleviated.

\section{JavaScript for Novice Programmers}

In this section, we first discuss why JavaScript seems to be a reasonable language to introduce computing to novices. We then show that JavaScript has several counterintuitive features that make it a poor choice for teaching beginning programmers. We then present RoboJS, a source-to-source compiler for JavaScript that transparently simplifies its semantics, which makes it behave like a "normal" language. Finally, we outline our end-user robotics library and how these pieces come together in the RoboJS IDE.

\subsection{Why JavaScript?}

There are several reasons why JavaScript is a good choice for an introduction to computing. First, JavaScript is one of the most widely used programming languages in the world (Ganesan 2019; TIOBE Software BV 2019; Stack Exchange, Inc. 2019), thus it introduces students to a technology that they are likely to encounter again. Second, there are several web-based IDEs for JavaScript that allow students to write and run programs within a web browser, without the need to install any further software. Finally, since JavaScript is a dynamically-typed language, there is no need to teach students how to work with a type-checker, which is necessary to program in Java or C.

\subsection{The Case Against JavaScript}

JavaScript has several peculiar features, which can confound novice and expert programmers (Maffeis, Mitchell, and Taly 2008; Guha, Saftoiu, and Krishnamurthi 2010). In this section, we present a few of these features and argue that they make JavaScript a poor choice for teaching programming.

No Arity Mismatch Errors JavaSript does not have aritymismatch errors, thus it is not an error to call a function with too many or too few arguments. If a function receives too many arguments, the extra arguments are silently dropped. If a function receives too few arguments, the elided arguments are set to the special value undefined. For example, our robot programming API has a moveTo function that takes three arguments: an $x$-coordinate, a $y$-coordinate, and an angle. We found that students frequently forget to supply the angle argument, and would call move To with only the coordinates. In most other languages, this omission would trigger an error (either during compilation or at runtime). However, no error occurs in JavaScript.

Implicit Type Conversions JavaScript performs many implicit type conversions under the hood. Whereas some type conversions can be valuable, others are very counterintuitive. For example, the relational operators of JavaScript convert all values to numbers, including functions and objects. Functions and objects are converted to NaN, which is a floating-point number, and all comparisons with NaN produce false. In our robot programming API there is a function called getBallPosX that takes zero arguments and returns the current position of the ball. Students would frequently forget to call zero-argument functions using parenthesis, and write code such as the following:

\section{if (robot.getBallPosX $>0)\{\ldots\}$}

The code above is wrong, since it is comparing the function, and not the result of calling the function. However, it does not raise an error. In other languages, including dynamically-typed languages such as Python, the equivalent code would raise an error. A mistake such as this is very hard to identify, even for experts.

Non-Existent Fields In JavaScript, it is not an error to access a non-existent field from an object. When a program does so, JavaScript returns the special value undefined. Worse, a program can explicitly set a field to the value undefined, which makes it even harder to discern if the 


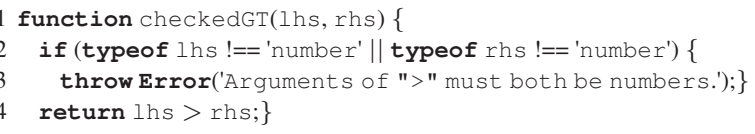

Figure 4: A simplified version of the runtime check for the greater-than operator in RoboJS.

field exists or not. Due to this behavior, simple spelling mistakes can produce undefined. In contrast, accessing nonexistent fields produces an error in Java (during compilation) and Python (at runtime).

\subsection{A Comprehensible Subset of JavaScript}

We develop a subset of JavaScript that we call RoboJS, which eliminates the problems listed above (among others). Moreover, our IDE transparently ensures that students' programs are in RoboJS, using a source-to-source JavaScript compiler that runs in the browser. The RoboJS compiler uses static checks when possible to catch errors before the program runs. When static checks are infeasible, it inserts dynamic checks that execute at runtime. Therefore, when a user uses a forbidden language feature (knowingly or not), it produces an error and aborts the program. The compiler is careful to ensure that its operation is transparent: the user never sees the inserted checks and the source locations are preserved so that the inserted checks do not affect how other errors are reported. Finally, since RoboJS is a strict subset of JavaScript, any RoboJS program runs outside the IDE.

For instance, we have seen that the comparison operators in JavaScript, such as the greater-than operator, perform unintuitive implicit type conversions. In contrast, RoboJS requires both arguments of the greater-than operator to be numbers. To do so, it replaces all occurrences of $x>y$ in students' code with a call to a library function that ensures that $x$ and $y$ are numbers (checkedGT $(x, y)$ in Figure 4). Note that students never see this function nor know that their code is being rewritten in this manner.

Similarly, in RoboJS, a function must receive exactly as many arguments as it declares. To accomplish this, the RoboJS compiler adds a check to the start of every function to assert that the number of actual arguments (arguments. length) is equal to the number of declared arguments. If not, RoboJS produces an error. Again, this check is transparent to the programmer.

RoboJS has several other checks and carefully constructed error messages, which shield students from JavaScript's peculiar features. In $\S 6$, we present evidence to show that RoboJS is effective and does help students catch mistakes early.

\subsection{Programming Robots with JavaScript}

In addition to shielding students from JavaScript's peculiar semantics, RoboJS also allows students to control robots using JavaScript. The RoboJS API is designed to gradually increase the amount of control that students have over the robots. For example, RoboJS provides several different

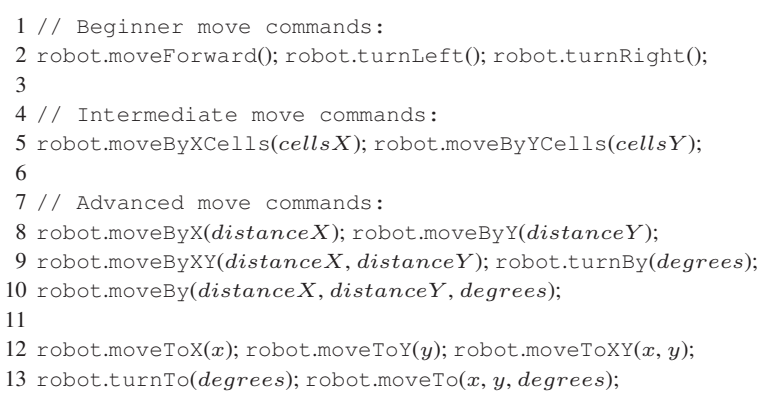

Figure 5: Progression of precision and detail with respect to how the students programmed robot movement.

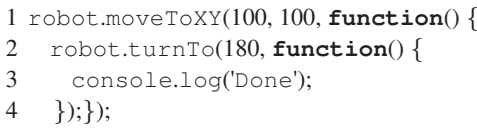

(a) Programming a robot using callback functions.

1 robot.moveToXY $(100,100)$;

2 robot.turnTo(180);

3 console.log('Done');

(b) Programming a robot with RoboJS.

Figure 6: JavaScript does not support blocking I/O, which can make it hard to write programs that communicate with any external service, including our robots. However, RoboJS simulates blocking $\mathrm{I} / \mathrm{O}$, which makes programming easier.

functions to move robots (Figure 5). At the beginning of the workshop, we introduced students to basic motion commands that moved the robots on a discrete grid (Figure 5, line 1). This allowed students to get comfortable typing JavaScript code, without the need to understand geometry in detail. Subsequently, we had students use functions that moved the robot by a given number of cells (Figure 5, line 4). This gave the students experience with function parameters and helped reduce repetitive coding. Finally, we presented movement in a continuous environment with angles of rotation, absolute motion, and relative motion (Figure 5, line 7). By gradually introducing students to more advance features as the workshop progressed, we made an effort not to overwhelm them with too much new content at once.

Another aspect of the library methods are the lack of data structures. Everything is a primitive value, both as input to the methods, and output from them. We only taught the students about numbers, booleans, and strings. For instance, instead of a single method to return the position of a robot in the continuous plane there were three; one each for the $x$ coordinate, $y$-coordinate, and angle. This API design allows us to avoid introducing arrays and objects.

\subsection{An In-Browser IDE and Runtime for RoboJS}

RoboJS includes an IDE that runs in a browser. The RoboJS IDE has a straightforward interface: 1) a handful of buttons, 
including buttons to start the program, stop the program, and open the robot simulator (section 3.4), 2) a list of files, 3) a code editor based on Monaco ${ }^{12}$, and 4) a REPL where students can view textual output and execute simple expressions immediately.

The RoboJS runtime system shields students from a key limitation of web browser APIs, which is that all network $\mathrm{I} / \mathrm{O}$ is non-blocking. Thus code that makes a network request must use a callback function to receive the response. Since our platform relies on the network to issue commands to robots, it appears that we need to teach callbacks and higherorder functions to have students do anything of interest. For example, if we wanted to have a robot first move to a location and turn only after the move command completes, we would have to teach students to write nested functions (Figure 6a). This style of programming is not suitable for beginners.

Instead, the RoboJS runtime allows students to write simple, straight-line programs (Figure 6b). Its APIs simulate blocking I/O on top of the web browser's non-blocking primitives by building on a tool called Stopify (Baxter et al. 2018). Stopify is a source-to-source JavaScript compiler that simulates multiple, cooperative threads of execution in JavaScript. ${ }^{13}$ Using Stopify, RoboJS maintains two logical threads: one thread runs the user's program and the other performs I/O operations. To perform an I/O operation, the user thread sends a message to the I/O thread, and then suspends itself. When the I/O operation completes, the I/O thread wakes the sleeping user thread and sends it the result.

\section{Computing and Robotics Workshop}

We ran a week-long workshop with twelve high-school students. The only prior programming experience in this group was that one student was familiar with TI-Basic and several had worked with Lego Mindstorms using a block-based language. Table 1 summarizes the curriculum and programming activities that we used over the course of the week. The curriculum incrementally introduces more sophisticated concepts in geometry, which was supported by the layers of abstraction in the RoboJS API. The students started every activity by programming in simulation. However, we encouraged them to frequently test their programs on physical robots (with modest supervision by the course staff). In the early activities that use discrete coordinates, programs reliably behave the same in simulation and on the real robots. However, with continuous coordinates and more sophisticated behaviors, such as catching and passing a ball, there are inevitable discrepancies between the simulation environment and the physical environment. This is an important lesson to learn when programming robots; it is necessary to frequently test programs on actual robots.

\section{Evaluation of RoboJS}

In this section, we evaluate the effectiveness of RoboJS by studying the kinds of errors that RoboJS caught during our workshop. We leverage data from our web-based IDE which

\footnotetext{
${ }^{12}$ https://microsoft.github.io/monaco-editor

${ }^{13}$ Technically, Stopify simulates first-class continuations, which we use to simulate cooperative threads (Wand 1980).
}

\begin{tabular}{|c|c|}
\hline Day & Summary \\
\hline 1 & $\begin{array}{l}\text { Topics: Introduction to the robots and program- } \\
\text { ming environment by example. Statements, vari- } \\
\text { ables, conditionals, and loops. Actuation, inputs, } \\
\text { and sensing. Activities: Mazes with multiple open } \\
\text { repetitive paths with and without dynamic obsta- } \\
\text { cles. }\end{array}$ \\
\hline 2 & $\begin{array}{l}\text { Topics: Discrete coordinate geometry and relative } \\
\text { motion. Functions. Planning and robot hardware. } \\
\text { Activities: Fixed item collection based on a ran- } \\
\text { dom robot location with and without adversarial } \\
\text { obstacles. }\end{array}$ \\
\hline 3 & $\begin{array}{l}\text { Topics: Continuous coordinate geometry and ab- } \\
\text { solute motion. Multi-agent robotics, navigation, } \\
\text { and more on planning (i.e. dynamic and adver- } \\
\text { sarial). Activities: Robot tag, both chasing and } \\
\text { avoiding agents to compete amongst each other } \\
\text { and against supplied programs. }\end{array}$ \\
\hline 4 & $\begin{array}{l}\text { Topics: More geometry, with special attention to } \\
\text { angles. Feature and API exposure needed to in- } \\
\text { teract with the ball and play soccer. Activities: } \\
\text { Penalty shootout, both goalie and striker agents to } \\
\text { compete amongst each other and against supplied } \\
\text { programs. }\end{array}$ \\
\hline 5 & $\begin{array}{l}\text { Topics: No new material. Activities: } 2 \mathrm{v} 2 \text { soccer } \\
\text { with } 4 \text { distinct roles; goalie, defender, primary and } \\
\text { secondary attacker. Students worked in teams to } \\
\text { write these agents, with elementary default code } \\
\text { provided. }\end{array}$ \\
\hline
\end{tabular}

Table 1: An overview of the topics and activities for each day of the workshop.

saves a history of edits for every file, so that students can easily revert to an older version if needed. Specifically, if a file has changed since the last run, then the IDE saves a copy of the program to the cloud when a user tries to run it again. Therefore, we expect that most revisions are programs that should have (partially) worked. We gathered 3,230 revisions total across ten student groups (i.e. accounts) over the course of one week. Table 2 summarizes the number of lines, revisions, files, and their sizes across all accounts.

\subsection{JavaScript Syntax Errors}

JavaScript reports syntax errors itself and our IDE gives a modicum of feedback: it highlights matching brackets and parenthesis. Therefore, we first checked the syntax of all revisions and found that $10 \%$ of the revisions had syntax errors (Table 3). We found far fewer errors on the last day of the workshop, which may be because students had gained experience or were modifying programs written earlier.

Most of the syntax errors we found involved missing or mismatched parentheses. Other common errors included writing else instead of else if, using an assignment operator directly before a block statement, and redeclared let-bound variables. 


\begin{tabular}{|r|r|r|r|r|r|}
\hline Account & Lines (L) & Revisions (R) & Files (F) & L/R & R/F \\
\hline 1 & 7,591 & 180 & 23 & 42.2 & 7.8 \\
\hline 2 & 6,478 & 208 & 14 & 31.1 & 14.9 \\
\hline 3 & 3,795 & 164 & 26 & 23.1 & 6.3 \\
\hline 4 & 7,871 & 296 & 25 & 26.6 & 11.8 \\
\hline 5 & 4,227 & 196 & 24 & 21.6 & 8.2 \\
\hline 6 & 5,253 & 194 & 18 & 27.1 & 10.8 \\
\hline 7 & 13,598 & 508 & 32 & 26.8 & 15.9 \\
\hline 8 & 18,796 & 462 & 29 & 40.7 & 15.9 \\
\hline 9 & 23,087 & 590 & 29 & 39.1 & 20.3 \\
\hline 10 & 15,472 & 432 & 17 & 35.8 & 25.4 \\
\hline Total & 106,168 & 3,230 & 237 & 32.9 & 13.6 \\
\hline
\end{tabular}

Table 2: An aggregate summary of program data per student account, which also shows the approximate program size (i.e. lines per revision) and number of edits (i.e. revisions per file).

\begin{tabular}{|r|r|r|r|}
\hline Account & Syntax Errors & RoboJS Errors & Revisions \\
\hline 1 & 10 & 82 & 180 \\
\hline 2 & 26 & 28 & 208 \\
\hline 3 & 6 & 12 & 164 \\
\hline 4 & 39 & 43 & 296 \\
\hline 5 & 18 & 46 & 196 \\
\hline 6 & 29 & 10 & 194 \\
\hline 7 & 22 & 60 & 508 \\
\hline 8 & 49 & 89 & 462 \\
\hline 9 & 80 & 54 & 590 \\
\hline 10 & 45 & 205 & 432 \\
\hline Total & 324 & 629 & 3,230 \\
\hline
\end{tabular}

Table 3: A count of JavaScript syntax errors and RoboJS runtime errors per student account. Note the RoboJS errors are estimated; see section 6.2 for context.

\subsection{Estimation of Errors Caught by RoboJS}

A post hoc analysis of the errors caught by RoboJS is less straightforward, because these programs cannot run without a (simulated) robot and the playing field in a particular configuration. Therefore, instead of counting the number of errors exactly, we conservatively estimate them by searching the text of all revisions for patterns that fail in RoboJS. For example, it is straightforward to check for arity-mismatch errors that involve RoboJS library functions, because we know their arity beforehand. In all, we wrote 340 regular expressions that represent patterns of code that RoboJS rejects and manually audited all matches to verify that they were valid. This approach is certain to produce false negatives and thus undercounts the effectiveness of RoboJS. On the other hand, false positives would only occur if students had unreachable code. In summary, $19 \%$ of the revisions had errors that RoboJS catches and JavaScript does not.

Table 4 breaks down the errors that RoboJS catches by category. The table has an example for each category, describes how JavaScript ordinarily behaves and the consequences of its behavior. In almost all cases, RoboJS catches the error early and halts the program with a sensible error message, instead of silently failing, which is what ordinary

\begin{tabular}{|c|c|c|}
\hline Pitfall & Count & Contrasting JavaScript and RoboJS \\
\hline $\begin{array}{l}\text { Loose comparison } \\
\text { Ex: true }==1\end{array}$ & 18 & $\begin{array}{l}\text { JS Behavior: Operand type coercion. } \\
\text { Consequence in JS: Potential false positives. } \\
\text { RoboJS Behavior: Not allowed. }\end{array}$ \\
\hline $\begin{array}{l}\text { Uninitialized vari- } \\
\text { able } \\
\text { Ex: let } x \text {; }\end{array}$ & 21 & $\begin{array}{l}\text { JS: undefined reference. } \\
\text { Consequence: Propagation of undefined (if } \\
\text { referenced before initialization). } \\
\text { RoboJS: Not allowed. }\end{array}$ \\
\hline $\begin{array}{l}\text { Conditional as- } \\
\text { signment } \\
\text { Ex: if }(x=0)\end{array}$ & 28 & $\begin{array}{l}\text { JS: Branches on value of RHS as a Boolean. } \\
\text { Consequence: Potential branching based on } \\
\text { non-Boolean literals. } \\
\text { RoboJS: Same branch behavior, but only al- } \\
\text { lowed if RHS evaluates to true or false. }\end{array}$ \\
\hline $\begin{array}{l}\text { Op type mismatch } \\
\text { Ex: 'x' } x^{\prime} 2\end{array}$ & 42 & $\begin{array}{l}\text { JS: NaN reference in the case of arithmetic/bit- } \\
\text { wise operators and shorthand assignment. } \\
\text { Consequence: Potential propagation of NaN. } \\
\text { RoboJS: Not allowed. }\end{array}$ \\
\hline $\begin{array}{l}\text { Arity mismatch } \\
\text { Ex: } \operatorname{set} X() \text {; }\end{array}$ & 213 & $\begin{array}{l}\text { JS: Treats elided parameters as undefined; } \\
\text { silently discards extra parameters. } \\
\text { Consequence: Propagation of undefined de- } \\
\text { pending on function implementation. } \\
\text { RoboJS: Only allowed for native JS functions. }\end{array}$ \\
\hline
\end{tabular}

Table 4: A comparison of behaviors with respect to a handful of beginner programming pitfalls.

JavaScript does.

Note that arity mismatch errors account for two-thirds of all errors caught by RoboJS. Moreover, two library methods accounted for nearly all the arity mismatch errors (particularly for account 10). As such, this suggests these methods were not properly explained or have misleading names, and thus presents an opportunity for improvement in the future.

To summarize, we find that when students ran their programs, they failed with syntax errors $10 \%$ of the time and with RoboJS errors 19\% of the time. These RoboJS failures are helpful to students, because without RoboJS, JavaScript simply fails silently and behaves in unintuitive ways. Therefore, we conclude that RoboJS helped students catch errors earlier than if we had used ordinary JavaScript.

\section{Future Work and Conclusion}

With some technological improvements and feature development, we plan to continue doing similar outreach workshops to further evaluate our methodology effectiveness.

It is also interesting to explore other avenues to utilize our existing platform. One idea is to use our system for future RoboCup team tryouts. Potential aspiring team members could have the opportunity to showcase their programming abilities and understanding of robotics principles through this higher-level medium. Should they exhibit proficiency in our environment, they can move onto the actual low-level $\mathrm{C}++$ development necessary for RoboCup. A second possibility would be the use of RoboJS in other teaching scenarios. Taking away the robotics library from RoboJS, reveals a strict subset of JavaScript that removes the hard edges of the language. This could be used for instructional purposes, and even general use, for better JavaScript programs and developer practice.

In conclusion, we present a means for teaching introductory computing and robotics principles with an industrystandard programming language and high-performance robots. This is made possible by careful learning abstractions coupled with safe and robust technology interfaces that 
are made available in a conducive environment.

\section{Acknowledgments}

This work was partially supported by NSF Awards CCF1717636 and IIS-1724101. Additional support was received from AFRL and DARPA agreement FA8750-16-2-0042. We thank Jarrett Holtz, Emily Pruc, and Sadegh Rabiee for their support in workshop preparation. We thank Emily Herbert and Donald Pinckney for their help running the workshop. We thank Andrew Pasquale and the HolyokeCodes team for their extensive help.

\section{References}

Balaban, D.; Fischer, A.; and Biswas, J. 2018. A real-time solver for time-optimal control of omnidirectional robots with bounded acceleration. In IEEE/RSJ International Conference on Intelligent Robots and Systems, 8027-8032.

Baxter, S.; Nigam, R.; Politz, J. G.; Krishnamurthi, S.; and Guha, A. 2018. Putting in all the stops: Execution control for JavaScript. In ACM SIGPLAN Conference on Programming Language Design and Implementation.

Biswas, J.; Mendoza, J. P.; Zhu, D.; Choi, B.; Klee, S.; and Veloso, M. 2014. Opponent-driven planning and execution for pass, attack, and defense in a multi-robot soccer team. In International Conference on Autonomous Agents and Multiagent Systems, 493-500.

Browning, B.; Bruce, J.; Bowling, M.; and Veloso, M. 2005. STP: Skills, tactics, and plays for multi-robot control in adversarial environments. Proceedings of the Institution of Mechanical Engineers, Part I: Journal of Systems and Control Engineering 219(1):33-52.

Bruce, J. R., and Veloso, M. M. 2006. Safe multirobot navigation within dynamics constraints. Proceedings of the IEEE 94(7):1398-1411.

Dee, H.; Cufi, X.; Milani, A.; Marian, M.; Poggioni, V.; Aubreton, O.; Rabionet, A. R.; and Rowlands, T. 2017. Playfully coding: Embedding computer science outreach in schools. In ACM Conference on Innovation and Technology in Computer Science Education.

Doran, M. V., and Clark, G. W. 2018. Enhancing robotic experiences throughout the computing curriculum. In $A C M$ Technical Symposium on Computer Science Education.

Estrada, F. J. 2017. Practical robotics in computer science using the LEGO NXT: An experience report. In ACM Conference on Innovation and Technology in Computer Science Education.

Featherston, E.; Sridharan, M.; Urban, S.; and Urban, J. 2014. DOROTHY: Enhancing bidirectional communication between a 3D programming interface and mobile robots. In AAAI Symposium on Educational Advances in Artificial Intelligence.

Ganesan, K. 2019. C\# or Java? TypeScript or JavaScript? machine learning based classification of programming languages. https://github.blog/2019-07-02-c-orjava-typescript-or-javascript-machine-learning-based- classification-of-programming-languages. Online; accessed 2019-09-1.

Gucwa, K. J., and Cheng, H. H. 2017. Making robot challenges with virtual robots. In ACM SIGCSE Technical Symposium on Computer Science Education.

Guha, A.; Saftoiu, C.; and Krishnamurthi, S. 2010. The essence of JavaScript. In European Conference of ObjectOriented Programming.

Holtz, J.; Guha, A.; and Biswas, J. 2018. Interactive robot transition repair with smt. In International Joint Conference on Artificial Intelligence (IJCAI), 4905-4911.

Kitano, H.; Suzuki, S.; and Akita, J. 2000. Robocup jr.: Robocup for edutainment. In Millennium Conference. IEEE International Conference on Robotics and Automation (ICRA), volume 1, 807-812.

Maffeis, S.; Mitchell, J. C.; and Taly, A. 2008. An operational semantics for JavaScript. In Asian Symposium on Programming Languages and Systems.

Magnenat, S.; Shin, J.; Riedo, F.; Siegwart, R.; and Ben-Ari, M. 2014. Teaching a core CS concept through robotics. In Innovation and Technology in Computer Science Education.

Martinez, C.; Gomez, M. J.; and Benotti, L. 2015. A comparison of preschool and elementary school children learning computer science concepts through a multilanguage robot programming platform. In ACM Conference on Innovation and Technology in Computer Science Education.

Musicant, D.; Laddha, A.; and Choi, T. 2017. Open-ended robotics exploration projects for budding researchers. In AAAI Conference on Artificial Intelligence.

Osborne, R. B.; Thomas, A. J.; and Forbes, J. R. 2010. Teaching with robots: A service-learning approach to mentor training. In ACM Technical Symposium on Computer Science Education.

Paramasivam, V.; Huang, J.; Elliott, S.; and Cakmak, M. 2017. Computer science outreach with end-user robotprogramming tools. In ACM SIGCSE Technical Symposium on Computer Science Education.

Paspallis, N.; Polycarpou, I.; Andreou, P.; Antoniou, J.; Kaimakis, P.; Raspopoulos, M.; and Terzi, M. 2018. An experience report on the effectiveness of five themed workshops at inspiring high school students to learn coding. In ACM Conference on Innovation and Technology in Computer Science Education.

2019. Stack overflow developer survey. https://insights. stackoverflow.com/survey/2019. Online; accessed 2019-0901.

2019. Tiobe index. https://www.tiobe.com/tiobe-index. Online; accessed 2019-09-01.

Wand, M. 1980. Continuation-based multiprocessing. In ACM conference on LISP and functional programming.

Weitzenfeld, A.; Biswas, J.; Akar, M.; and Sukvichai, K. 2015. Robocup small-size league: Past, present and future.

Zickler, S.; Laue, T.; Jr., J. A. G.; Birbach, O.; Biswas, J.; and Veloso, M. 2014. Five years of ssl-vision-impact and development. 